\title{
Densidad óptica de contenido gástrico en la valoración rápida de la madurez pulmonar del recién nacido
}

\author{
Liliana Brandell T. ${ }^{1}$; Waldo H. Sepúlveda L. ${ }^{2}$; \\ Heriberto Araneda $C^{2}$; Mónica Mangiamarchi $\mathbf{F}^{3}$

\section{Rapid assessment of pulmonary matarity by optical density in newborn's gastric aspirate}

\begin{abstract}
The authors studied the correlation between optical density at $650 \mathrm{~nm}\left(O D_{650}\right)$ in paired samples of amniotic fluid and neviborn gastric aspirate obtained from 50 pregnant women who underwent cesarean defivery and from their respective newborns. There was a good correlation between both samples ( $r=0.92)$, which demonstrates that $\mathrm{OD}_{6} \mathrm{~s}$ is a reliable method for studying neonatal pulmonary maturity, especially in those high.risk cases in which no prenatal study is available. None of the infants with mature readings (OD $s_{s}>0.10$ ) and 6 out of 13 infants with immature readings $\left\{O D_{6}\right.$ so $<0.10$ ) developed $R D S$.

(Key words: newborn, gastric aspirate, amniotic fluid, opt ical density, pulmonary surfactant.)
\end{abstract}

La determinación del grado de madurez pulmonar fetal (MPF) en líquido amniótico (LA) es un procedimiento ampliamente utilizado en el manejo del embarazo de alto riesgo ${ }^{1,2}$. Sin embargo, existen situaciones clínicas en que es difícil contar con LA para su análisis, como por ejemplo ruptura prematura de membranas, parto prematuro inminente o emergencia maternofetal que requjere interrupción inmediata del embarazo. En estos casos cobra especial importancia el análisis del contenido gástrico (CG) del recién nacido ( $R N)$ obtenido inmediata. mente después del parto, ya que este fluido biológico corresponde a LA deglutido in tutero por el feto ${ }^{3}$.

Entre los exámenes practicados en CG para evaluar madurez pulmonar en el $\mathrm{RN}$ existe experiencia clínica con la prueba de Clements ${ }^{4,5}$, el índice lecitina/esfingomielina ${ }^{6-8}$ y la detección de fosfatidilglicerol ${ }^{9}$. Aunque la lectura de la densidad óptica a $650 \mathrm{~nm}\left(\mathrm{DO}_{650}\right)$ ha sido ampliamente utilizada cono método rápido para

1. Programa de especialización en Perina tologia. Facultad de Medicina, Universidad de Concepción.

2. Servicio y Departanento de Obstetricia y Ginecologia. Hospital Clínico Regional Guillermo Grant Benavente, Concepción.

3. Programa de Especialización en Enfermería Pediátrica. Facultad de Medicina, Universidad de Concepción. evaluar MPF durante el embarazo ${ }^{1,2,10,11}$ no existen referencias en la literatura sobre su rendimiento en CG de RN. El propósito de este trabajo fue estudiar la correlación entre la $\mathrm{DO}_{650}$ de CG de RN obtenido en los primeros minutos de vida y la $\mathrm{DO}_{650}$ de LA obtenido inmediatamente antes del parto. Se analiza además la capacidad de este examen para predecir la presencia de síndrome de dificultad respiratoria (SDR) en el periodo neonatal.

\section{Material y Métodos}

Se ostudió en forma prospectiva 50 pacientes cuyo parto se verificó por operación cesárea en el Servicio de Obstetricia y Ginecologia del Hospital Guilhermo Grant Benavente de Concepción. En todas ellas se obtuvo muestras pareadas de L.A y de CG del RN no contaminadas ni con sangre ni con meconio según los procedimientos previamente comunicados por los autores ${ }^{5}$. El LA se obturo mediante punción directa de las membranas orulares después de la histerostomía y antes de la amniotomía, y el CG mediante pipeta de De Lee después de introducir sonda orofaringea a la cavidad gástrica del $\mathbf{R N}^{5}$. Las muestras se transportaron inmediatamente al laboratorio en frasca de vidrio, donde fueron procesadas por el mismo profesional (HA) de acuerdo al siguiente protocolo: medición del grado de acidez con papel indicador $\mathrm{pH}$; homogeneización por agitación en aparato Vortex, centrifugación a $3000 \mathrm{rpm}$ por $5 \mathrm{~min}$, y lectura espectrofotométrica del sobrenadante a $650 \mathrm{~nm}$, usando tnetanol como 
blanco ${ }^{11}$. E1 grado de asociación entre ambas variables se estudió a través del coeficiente de correlación y regresión lineal, utilizando un programa estadístico STAT (Sharp Scientific Calculator EL-5103, Osaka, Japón).

De acuerdo a nuestros estudios previos ${ }^{11}$, se consideró que lecturas de $\mathrm{DO}_{6}$ so $>0.10$ indjcaban madurez pulmonar y lecturas $<0,10$ indicaban inmadurez pulmonar. Los RN fueron seguidos hasta su alta hospitalaria, dando especial importancia al registro de SDR, cuyo diagnóstico y tratamiento se realizó en la Unidad de Neonatología de acuerdo a los criterios clínicos y radiológicos correspondientess.

\section{Resultados}

Todas las muestras obtenidas, tanto de LA como de CG tuvieron $\mathrm{pH} \geqslant 6$. Los valores de $\mathrm{DO}_{650}$ en LA y CG y la correlación entre ambos se muestra en la figura 1 , observándose alta correlación entre ambas muestras $(r=0,92)$. Trece RN (26\%) tuvieron lecturas de $\mathrm{DO}_{650}$ $<0,10$ y $37(74 \%)$ tuvieron lecturas de $\left.\mathrm{DO}_{650}\right\rangle$ 0,10 . Con respecto a la predicción de madurez pulmonar, níngún $\mathrm{RN}$ con $\mathrm{DO}_{650} \geqslant 0,10$ en CG presentó SDR (positivos verdaderos, $100 \%$; positivos falsos, $0 \%$ ). En cambio, 6 de $13 \mathrm{RN}$ con $\mathrm{DO}_{650}<0,10$ presentaron SDR (negativos verdaderos $46 \%$; negativos falsos $54 \%$ ), 3 de los cuales correspondieron a enfermedad de membrana hialina.

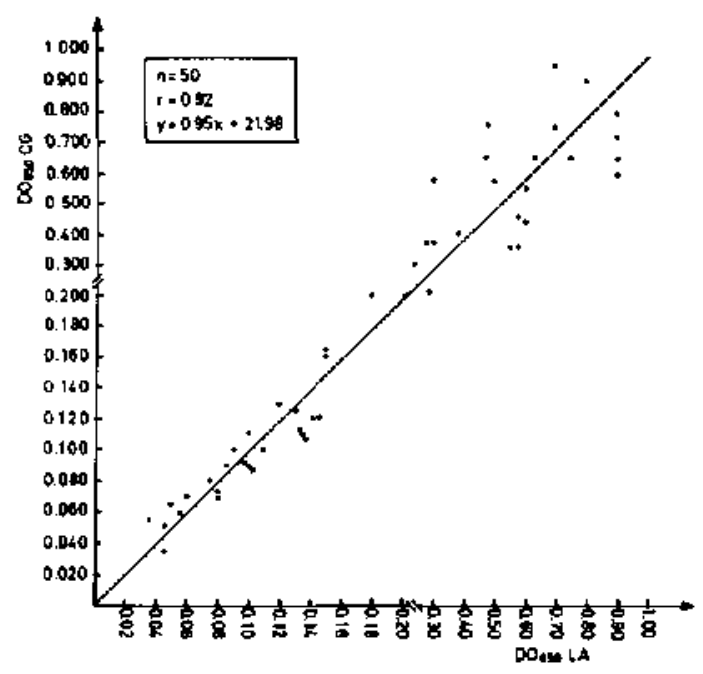

Figura 1: Correlación entre la densidad óptica a 650 $\mathrm{nm}\left(\mathrm{DO}_{650}\right)$ en contenido gástrico $(\mathrm{CG})$ de recién nacido y líquido amniótico (LA).

\section{Comentario}

La lectura de la $\mathrm{DO}_{650}$ en LA es un examen rápido para valorar el grado de MPF, que tiene la ventaja de requerir equipos poco complejos disponibles en cualquier laboratorio y permite, a diferencia de la prueba de Clements, medición cuantitativa de la $\mathrm{MPF}^{\mathrm{l}, 2,10,1 t}$. Se basa en la determinación espectrofotométrica de la turbidez del LA resultante de la presencia de cuer. pos lamelares, esferas de 1 a $2 \mu$ que correspon. den al surfactante alveolar en suspensión $y$ aumentan a medida que el feto madura ${ }^{10-12}$.

Nuestro estudio demuestra que la $\mathrm{DO}_{650}$ en CG de $\mathrm{RN}$ tiene buena correlación con la $\mathrm{DO}_{6 s 0}$ en LA $(\mathrm{r}=0,92)$, lo que indica que el CG obtenido inmediatamente después del nacimiento es un fluido tan confiable como el LA obtenido durante el embarazo para predecir madurez pulmonar. Al utilizar los mismos valores de corte para predecir madurez pulmonar que en $\mathrm{LA}^{11}$ no tuvimos resultados positjos falsos con lecturas de $\mathrm{DO}_{650} \geqslant 0,10$ en $\mathrm{CG}$, hecho que permite asegurar que el $\mathrm{RN}$ no presentará SDR durante el periodo neonatal. Esto tiene importancia práctica, ya que en caso de prematuridad permite manejar al $\mathrm{RN}$ en unidades de menor complejidad y a cargo de personal menos entrenado, evitando traslados innecesarios. Asimismo, ayuda al clinico en el diagnóstico diferencial del SDR, ya que de presen. tarse en un RN con lectura sugerente de madurez resulta poco probable que se trate de enfermedad de membrana hialina ${ }^{5,13}$. Por el contrario, encontramos un alto número de resultados negativos falsos, ya que 7 de $13 \mathrm{RN}$ con keturas $<0,10$ no presentaron SDR y de $\operatorname{los} 6$ que lo presentaron sólo $3 \mathrm{RN}$ correspondieron a enfermedad de membrana hialina. En este sen. tido la $\mathrm{DO}_{650}$ en CG del RN tiene alta sensibilidad para predecir madurez, pero baja especifjci. dad para predecir inmadurez ${ }^{11,14}$, hecho que comparte con la mayoría de los exámenes utilizados en la valoración de la MPF ${ }^{1,2}$.

Al analizar los casos en forma individual encontramos que algunas muestras tuvieron difeIencias importantes entre la lectura del LA y la del CG. Estas muestras se agruparon en lecturas $>0,50$, es decir, altamente opalescentes, por lo que no influyeron en el resultado neonatal al corresponder a casos categóricamente maduros. Debe tenerse presente que en este tipo de muestras los filamentos mucosos producto de la secre- 
ción gástrica del RN pueden originar resultados negatjvos falsos ${ }^{15}$, por lo que consideramos que la homogeneización de la muestra es un paso im. portante dentro del procedimiento, ya que durante la posterior centrifugación tanto los filamentos de mucus como el vérnix sedimentan, resultando un sobrenadante en que la opalescencia refleja con mayor precisión la presencia de surfactante en suspensión y no la de otro material.

Nuestros resultados nos permiten proponer la utilización de la lectura de la $\mathrm{DO}_{650}$ en $\mathrm{CG}$ como un método rápido, simple y de bajo costo para la valoración de la madurez pulmonar en e] periodo neonatal, lo que es especialmente útil en el manejo de RN con riesgo de desarrollar SDR o como parte del estudio en el diag. nóstico diferencial de estos cuadros. La implementación de este examen en las salas de atención inmediata del RN permite contar con un método más objetivo que la prueba de Clements en la evaluación de la madurez pulmonar neo. natal.

\section{Resumen}

Se estudió la correlación entre la densidad óptica a $650 \mathrm{~nm}\left(\mathrm{DO}_{650}\right)$ en 50 muestras pareadas de liquido amniótico (LA) y contenido gástrico (CG) de recién nacido (RN) provenientes de igual número de embarazadas y respectivos RN. Las muestras de LA fueron obtenidas durante operación cesárea inmediatamente antes del nacimiento y las de CG en los primeros minutos de vida. Se encontró buena correlación entre ambas muestras $(\mathrm{r}=0,92)$, lo que demuestra que la lectura de la $\mathrm{DO}_{650}$ en CG es un método igualmente confiable que la realizada en LA para estudiar la madurez pulmonar. Este método es especialmente útil en aquellos RN con alto riesgo de presentar síndrome de dificultad respiratoria (SDR) en los que no se cuenta con estudio prenatal y como parte del estudio en el diagnóstico diferencial de estos cuadros. Ninguno de los $37 \mathrm{RN}$ con lecturas maduras $\left(\mathrm{DO}_{650}\right.$ $\geqslant 0,10)$ y 6 de $\operatorname{los} 13 \mathrm{RN}$ con lecturas inmaduras $\left(\mathrm{DO}_{650}<0,10\right)$ presentaron SDR.
(Palabras clave: recién nacido, aspirado gástrico, liquido amniótico, densidad ôptica, surfactante pulmonar.)

\section{Referencias}

1. Weiner $S A$, Weinsfein $L$ : Fetal pulmonary maturity and antenatal diagnosis of respiratory distress syndrome. Obstet Gynecol Surv 1987; 42: 75-81.

2. Garite TS: Fetal maturity testing. Clin Obstet Gynecol 1987; 30: 985.991.

3. Avery $G B$, Randolph $J G$, Weaver $T$ : Gastric acidity in the first day of life. Pediatrics $1966 ; 37: 1005$ 1007.

4. Evans fJ: Prediction of respiratory-distress syndrome by shake test on newborn gastric aspirate. N Engl J Med 1975; 292: 1113-1115.

5. Brandell L. Sepulveda W. Araneda $H$ : Prueba de Clements en contenido gástrico de recién nacidos en la predicción de madurez pulmonar. Rev Chil Pediatr 1990; 61:299-302.

6. Borer $R C$, Kuths $I R$, Holt $I F$ et al: Accuracy of gastric aspirate lecithin/sphingomyelin ratio and chest roentgenogram in the diagnosis of RDS. Pediatr Res $1974 ; 8: 444$.

7. Amstrong D, Van Wormer DE, Gideon WP: Predicting respiratory distress by thin-layer chromatography of the newborn gastric aspirate. Obstet Gynecol 1976; 48:35*39.

8. Korvenranta $H:$ Gastric aspirate lecithin/sphingomyelin ratio and neonatal breathing difficulties. Gynecol Obstet Invest 1983; 15: 177-184.

9. Schmidt-Sommerfeld E. Kattner $\boldsymbol{W}$, Penn D: The role of phosphatidylglycerol in phospholipid analysis of tracheal and gastric aspirate in premature infants. J Perinat Med 1987; 15; 31-36.

10. Sbarra AJ, Michlewitz $H$, Selvaraj $R J$ et al.: Relation between optical density at $650 \mathrm{~nm}$ and $\mathrm{L} / \mathrm{S}$ ratios. Obstet Gynecol 1977; 50: 723.724.

11. Sepúlvedo WH, Araneda $H$, Brandell $I$, Contreras $V$, Varela $J$ : Evaluación de la madurez pulmonar fetal por la densidad óptica del líquido amniótico y prueba de Clements modificada. Rev Chil Obstet Ginecol 1988; 53: 261-267.

12. Sbarra AJ, Cetrilo $C L$, Selvaraj RJ et al.: Surfac. tants, L/S ratio, amniotic fluid optical density and fetal pulmonary maturity. J Reprod Med $1982 ; 27: 34-38$.

13. Lewins MS: The gastric-aspirate shake test and group B streptococcal disease. N Engl J Med 1978; 298: 1200 .

14. Aroneda $H$, Sepúlveda $W H$, Vlera $E:$ Densidad óptica a $650 \mathrm{~nm}$ y fosfatidiglicerol en la evaluación de la inmadurez pulmonar fetal. Rev Chil Obstet Ginecol 1991; 56: 104-106.

15. Gebhordt DOE. Ruys $J H$ : A pitfall in the shake test on gastric aspirate. N Engl I Med 1976; 294: 225 . 